\title{
A plausible mechanism for a non-pharmacological therapy for hypertension
}

\author{
Jack M. Gorman
}

Received: 20 November 2014 / Accepted: 11 January 2015/Published online: 11 February 2015

(C) Springer-Verlag Berlin Heidelberg 2015

One of the triumphs of modern medicine and public health has been the recognition that high blood pressure is a risk factor for cardiovascular disease and the subsequent development of multiple effective pharmacological interventions to lower blood pressure in hypertensive patients.

None of these medications is, of course, without adverse side effects. Because autonomic nervous system (ANS) dysfunction is suspected as an important cause of hypertension there is long-standing interest in the possibility that behavioral interventions that affect the ANS might also be effective for treating hypertension, with fewer risks. Modesti et al. provide us with impressive evidence that this may indeed be possible. It was previously shown that daily sessions of slow breathing significantly lowered 24-h ambulatory blood pressure in patients with mild hypertension, an effect that was sustained for 6 months after breathing sessions had been discontinued. Here they sought to understand whether the ameliorative effect of slow breathing on blood pressure is accompanied by measureable changes in ANS function. In a controlled study, they indeed found that 1 week of music-guided slow breathing sessions lasting at least $30 \mathrm{~min}$ each increased the high frequency component of heart period variability and reduced renal resistance. These changes preceded improvement in both baroreflex sensitivity and blood pressure.

The findings are important because they not only add to mounting evidence that behavioral interventions like meditation, yoga, and slow breathing can improve cardiovascular and immune function but also provide a plausible mechanism by which slow breathing improves blood

J. M. Gorman $(\bowtie)$

Franklin Behavioral Health Consultants, New York, USA

e-mail: jackgorm@gmail.com pressure in patients with hypertension. Autonomic imbalance, characterized by excessive sympathetic and deficient parasympathetic innervation to the heart, has long been linked to risk for cardiovascular disease [1]. Interestingly, patients with depression and several forms of anxiety disorder have evidence of reduced heart rate variability. Others and we have shown that effective cognitive behavioral therapy for patients with panic disorder also results in an increase in high frequency heart period variability, representing an improvement in parasympathetic control over cardiovascular physiology [2]. Thus the well-known links between psychiatric illness and risk for heart disease may be similarly mediated at least in part by ANS imbalance and be susceptible to behavioral interventions.

As Modesti et al. recognize, this is a small sample study that requires replication. It will also be interesting to find out how long these positive ANS changes persist once slow breathing sessions are discontinued. It may at first be difficult to understand how they could be sustained in the absence of an ANS manipulation like slow breathing, but it must also be remembered that the effect on blood pressure seems to last at least 6 months after treatment discontinuation. The ultimate test of slow breathing efficacy will be comparison of antihypertensive medication to behavioral interventions in randomized controlled trials. This will enable us to understand whether the magnitude of the effect of slow breathing approaches that of pharmacological treatment. Finally, longterm studies to assess whether slow breathing and other behavioral interventions effectively lower cardiovascular disease risk will be required.

It is also unclear as to what extent hypertensive patients will adhere to $30 \mathrm{~min}$ of daily slow breathing exercises. Adherence in this study was excellent, but the 37 participants were a subgroup of 243 patients who 
presented to the clinic with hypertension. Still, having an effective treatment that does not have adverse side effects should be an important development in the treatment of high blood pressure and the reduction of risk for serious heart disease. Moreover, understanding the physiological method by which slow breathing lowers blood pressure should stimulate more work in this fascinating and important area.

\section{References}

1. Thayer JF, Yamamoto SS, Brosschot JF (2010) The relationship of autonomic imbalance, heart rate variability and cardiovascular disease risk factors. Int J Cardiol 141:122-131

2. Garakani A, Martinez JM, Aaronson CJ, Voustianiouk A, Kaufmann H, Gorman JM (2009) Effect of medication and psychotherapy on heart rate variability in panic disorder. Depress Anxiety 26:251-258 